\title{
Cardiac Tamponade: An Acute and Subacute Clinical Challenge
}

\author{
Kara Stout ${ }^{1}$, Jennifer Forbes ${ }^{1}$, Stephanie Cochran ${ }^{2}$, Hussein Al-Mohamad ${ }^{2}$, Merrill Krolick ${ }^{2}$, Ronald Walsh ${ }^{2}$ \\ ${ }^{1}$ Department of Internal Medicine, Largo Medical Center, Largo, Florida \\ ${ }^{2}$ Department of Cardiology, Largo Medical Center, Largo, Florida
}

${ }^{\star}$ Corresponding Author: Kara Stout, Department of Internal Medicine, Largo Medical Center, Largo, Florida; E-mail: kstout@atsu.edu

Received: November 26, 2019; Accepted: November 30, 2019; Published: December 26, 2019

\section{Background}

Pericardial disease resulting in a pericardial effusion is a common clinical finding with numerous etiologies identified including trauma, infection, neoplasm, autoimmune etiology, metabolic cause, or a drug-related process. Once the diagnosis of pericardial effusion has been made, it is important to determine whether the effusion is creating significant hemodynamic compromise resulting in cardiac tamponade. In addition, the timing of accumulation of the pericardial fluid significantly affects the presentation of each case. Typically, a rapid accumulation is seen in acute cases and a delayed accumulation in a subacute presentation. Distinguishing between the diagnoses of pericardial effusion and cardiac tamponade is vital in order to treat a patient appropriately. We report two cases of pericardial effusion with differing etiologies, both resulting in cardiac tamponade physiology and exhibiting components of Beck's triad. Not only did the two cases differ in etiologies, but in the length of fluid accumulation and timeframe of presentation as one presented as a rapidly accumulating pericardial effusion with acute cardiac tamponade and the other as subacute. Despite these differences, both cases were managed similarly with the creation of a pericardial window and drainage of the pericardial fluid to restore normal hemodynamics.

\section{Case series}

Case \#1: An 80 year-old Caucasian male with a past medical history of a non-ischemic cardiomyopathy with a left ventricular ejection fraction of 35\% and New York Heart Association Class III, left bundle branch block, hypertension, hyperlipidemia, abdominal aortic aneurysm, and previous cerebrovascular accident presented for routine cardiac resynchronization therapy defibrillator (CRT-D) placement. The left pectoral area was sterilely prepped and the left subclavian vein accessed. A defibrillator lead was fixated with a screwin technique in the apical septal location of the right ventricle (RV); the lead was repositioned twice due to inappropriate parameters. An atrial-pacing electrode was then advanced and fixed with a similar screw-in technique in the high right atrium. Next, a coronary sinus guiding catheter was advanced into the coronary sinus and into a posterolateral branch. A left ventricle (LV) pacing lead was then advanced to the posterolateral branch; both the guidewire and the lead terminated prematurely in the lateral wall, suggesting a suboptimal position for optimal CRT. The guide catheter was then advanced into a second lateral wall vessel, and then the pacing lead was successfully placed in the mid lateral wall of the LV. All three leads tested adequately and optimized without extracardiac stimulation noted. The leads were connected to a CRT-D generator, and then the pocket was closed. Thirty minutes postoperatively, the patient's blood pressure dropped to $92 / 60 \mathrm{mmHg}$ with a heart rate of $88 \mathrm{bpm}$. The patient was in no visible distress, but mild jugular venous distention and muffled heart sounds were noted on physical exam. Stat transthoracic echocardiogram (TTE) demonstrated a moderate pericardial effusion conferential to the heart, with RV diastolic collapse and respirophasic changes of less than $50 \%$ variation in the inferior vena cava, suggesting mild cardiac tamponade (Figure1).

Cardiothoracic surgery took the patient directly to the operating room, and an emergent cardiac window for suspected RV perforation was completed. Although no clear perforation was seen interoperatively; $175 \mathrm{~mL}$ of blood was evacuated with improvement in systolic blood pressure to over $150 \mathrm{mmHg}$. A chest tube was placed and set to suction with an additional $500 \mathrm{ml}$ of blood removed over 48 hours. The patient recovered and was discharged with close cardiology follow up.

Case \#2: A 66 year-old African American male with a past medical history of small cell lung cancer, COPD, tobacco use, and occupational exposure to asphalt presented with a one-day history of a new, tender right clavicular mass and worsening pleuritic chest pain and dyspnea with exertion. The patient was initially diagnosed with a small cell lung cancer in July 2018 . He was initially treated with palliative chemotherapy including Etoposide and Cisplatin, and he achieved remission in October 2018. Unfortunately, he subsequently developed a recurrence in the left upper lobe in January 2019. The patient received palliative radiation until he was unable to tolerate treatments. Further mediastinal and osseous metastases were found after the chemotherapy and radiation were discontinued. Nine days prior to this presentation to the hospital, the patient underwent a therapeutic left thoracentesis due to a symptomatic, malignant pleural effusion. On primary assessment in the emergency room, the patient was found to be tachycardiac with a pulse of $121 \mathrm{bpm}$ and oxygen saturation of $94 \%$ breathing ambient air. The physical examination revealed a large, $7 \times 6 \mathrm{~cm}$ right clavicular mass extending into the neck. A cardiopulmonary exam revealed sinus tachycardia, jugular venous distention, and decreased breath sounds on the left. An EKG showed 
sinus tachycardia, a low voltage QRS, and no significant ischemic changes. A chest $\mathrm{x}$-ray and then stat non-contrast CT neck/thorax revealed a new, large pericardial effusion and left greater than right bilateral pleural effusions (Figures 2, 3). Cardiology was consulted and a stat TTE demonstrated a large pericardial effusion, anterior and posterior to the heart, with features consistent with tamponade physiology (Figure 4).

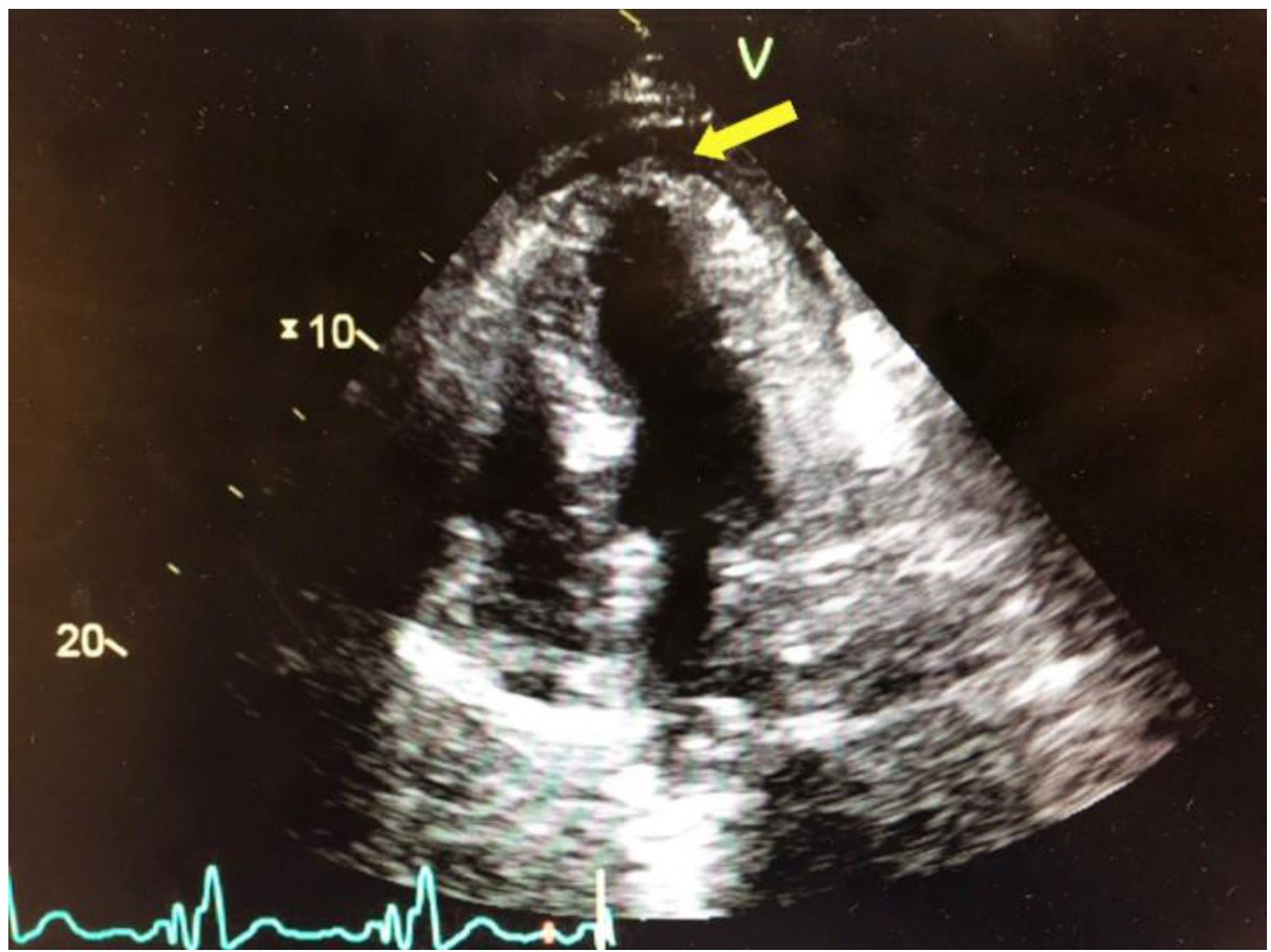

Figure 1. Acute cardiac tamponade with a moderate pericardial effusion anterior and posterior to the heart, with partial right atrial and right ventricular collapse.

Cardiothoracic surgery was then consulted and a therapeutic pericardial window was performed with $700 \mathrm{cc}$ of bloody fluid drained. In addition, a portion of the pericardium was resected and sent to pathology. Cytology revealed a malignant pericardial effusion consistent with small cell carcinoma. In addition, the patient underwent left thoracentesis and placement of a chest tube intra-operatively. He tolerated the procedures well and was transferred out of the ICU to the medical-surgical floor the day following surgery. Two days later, he underwent a right thoracentesis for a symptomatic, right-sided pleural effusion. On post-operative day 5 , the patient acutely decompensated and was transferred to the ICU. Vital signs upon transfer showed a blood pressure of $67 / 51 \mathrm{mmHg}$, heart rate of $118 \mathrm{bpm}$, respiratory rate of 45 , and pulse oximetry of $60 \%$. An arterial blood gas was obtained that showed $\mathrm{pH}$ of $7.153, \mathrm{pCO} 2$ of $54.7, \mathrm{pO} 2$ of 29 , and bicarbonate of 19.2. A stat EKG and TTE were obtained, with the EKG revealing atrial fibrillation with rapid ventricular rate, premature ventricular or aberrantly conducted complexes, and a low voltage QRS. Bedside TTE revealed a recurrent, circumferential, small pericardial effusion without signs of tamponade or RV strain. In addition, no recurrence of left pleural effusion was identified. Due to the patient's condition, the patient's family made the decision to proceed with comfort measures only and further hospice care. Unfortunately, the patient passed away on post-operative day 7 due to complications related to the underlying small cell carcinoma and the associated malignant pleural and pericardial effusions. 


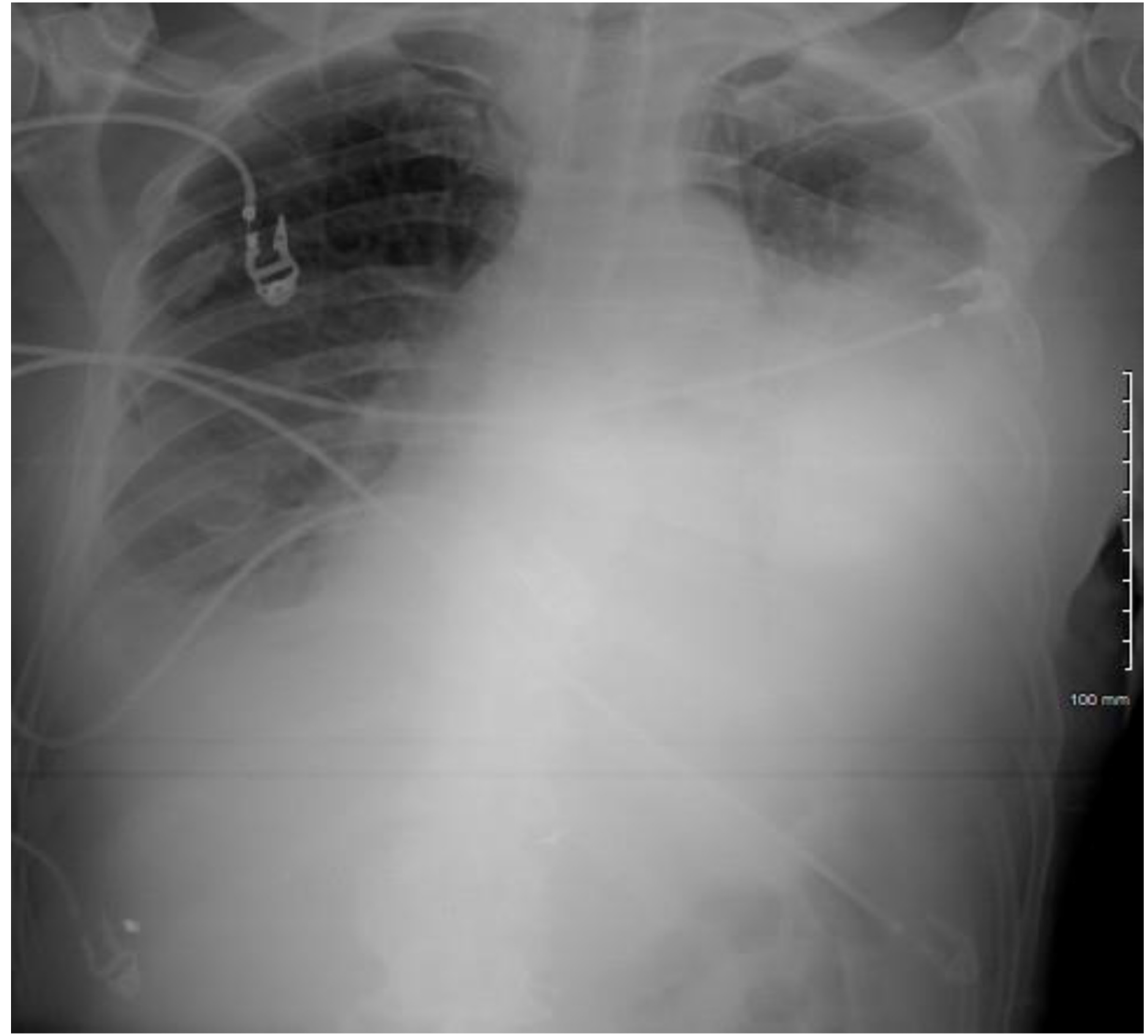

Figure 2. Chest X-ray revealing a flask-shaped cardiac silhouette as evidenced in pericardial effusions, as well as a large, left-sided pleural effusion. 


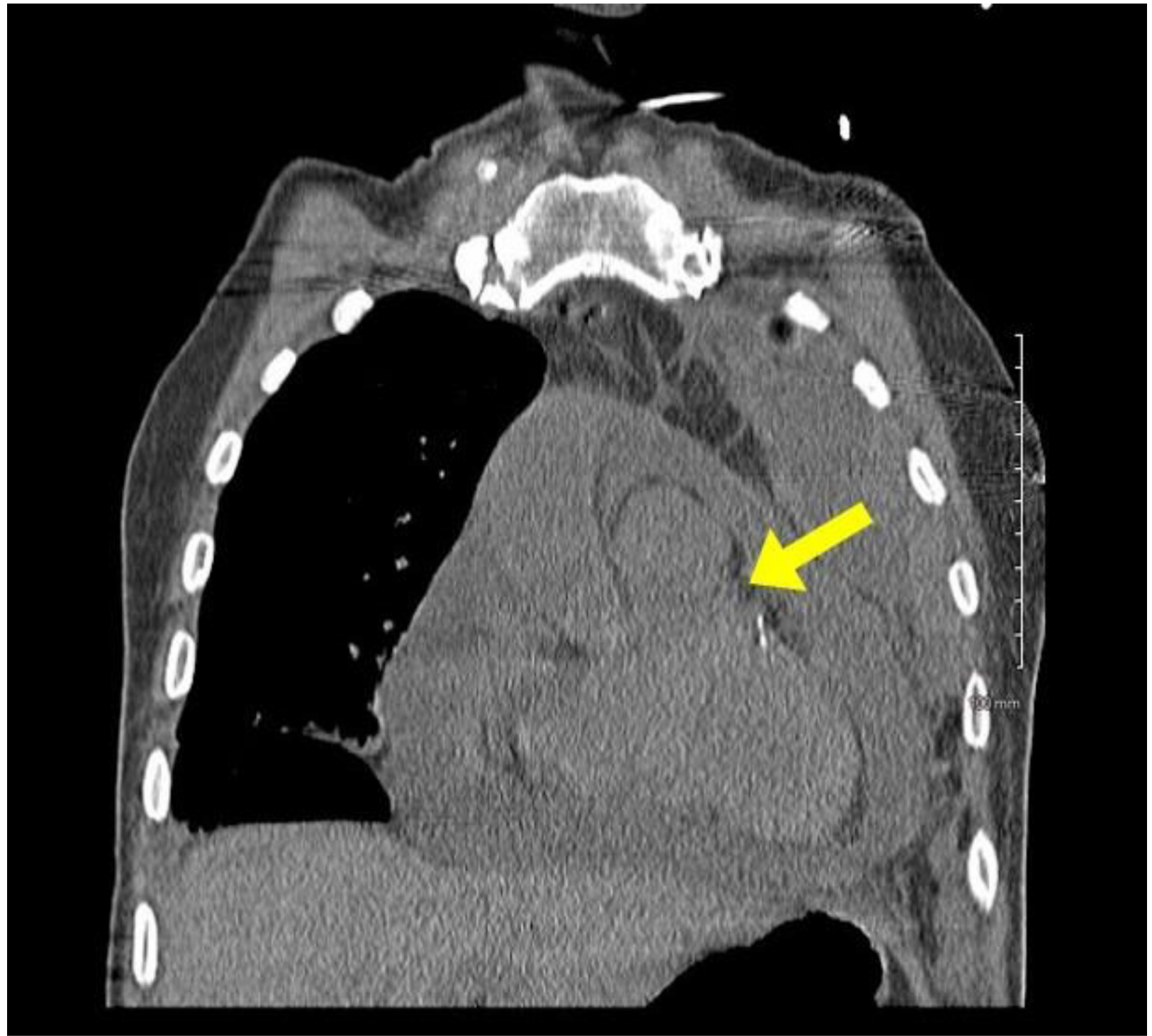

Figure 3. Stat $\mathrm{CT}$ chest with a coronal view demonstrating a large pericardial effusion.

\section{Discussion}

The presentation of cardiac tamponade depends on the timing over which the pericardial effusion accumulates. Acute cardiac tamponade occurs within minutes, and this physiology is seen in our first case. It resembles cardiogenic shock, and an emergent reduction in intrapericardial pressure (IPP) is required for treatment [1]. The second type, subacute cardiac tamponade, occurs over days to weeks. This is seen in our second case. Patients initially may be asymptomatic until the IPP reaches a critical limit, at which time symptoms such as chest pain or dyspnea ensue [1]. The pericardium is a fibroelastic sac that encases the heart and proximal great vessels [2]. It is composed visceral and fibrous parietal layers, typically containing $50 \mathrm{ml}$ or less of serous fluid which serves as a lubricant to reduce the friction on the epicardium [2,3]. Any processes causing this volume to exceed the typical amount, thus raising the IPP, is known as a pericardial effusion [2]. Pericardial effusions are classified based upon onset, size, location, hemodynamic changes, and composition [3]. Pericardial effusions can be loculated or circumferential and can be composed of transudative fluid, exudative fluid, pus, air, or blood [3]. Inflammatory causes can include viral, bacterial, fungal, or protozoal infections. A pericardial effusion can also be related to autoimmune disease, drug hypersensitivity, or post cardiac procedure syndromes as seen in our first case [2]. Non-inflammatory causes include hypothyroidism, trauma, or a reduction in lymphatic drainage from heart failure, cirrhosis, nephrotic syndrome, or malignancy [2] as seen in the second case.

Under normal physiologic conditions the pericardial pressures are low, and systolic and diastolic changes cause little interventricular interaction. Inspiration causes a decreased pulmonary vascular resistance, leading to an increase in venous return to the right ventricle and a small drop in the pulmonary capillary wedge and left ventricular end diastolic pressures. This leads to a decrease in systolic blood pressure of approximately $5 \mathrm{mmHg}$ [2]. An increase in the fluid collection in the pericardial space causes right and left ventricular pressures to increase and equalize. In addition, with the normal increasing pressure in the right ventricle with inspiration, 
the rigid pericardium prevents the free wall from expanding, leading to bulging of the interventricular septum into the left ventricle [2]. During inspiration in a patient with cardiac tamponade, the drop in pulmonary venous pressure leads to a drop in left atrial and pulmonary capillary wedge pressures. The left ventricular diastolic pressure remains elevated due to a leftward bowing of the interventricular septum and reduced left ventricular compliance. This exacerbates a decline in left ventricular filling pressures, and ultimately leads to a decrease in stroke volume [2].

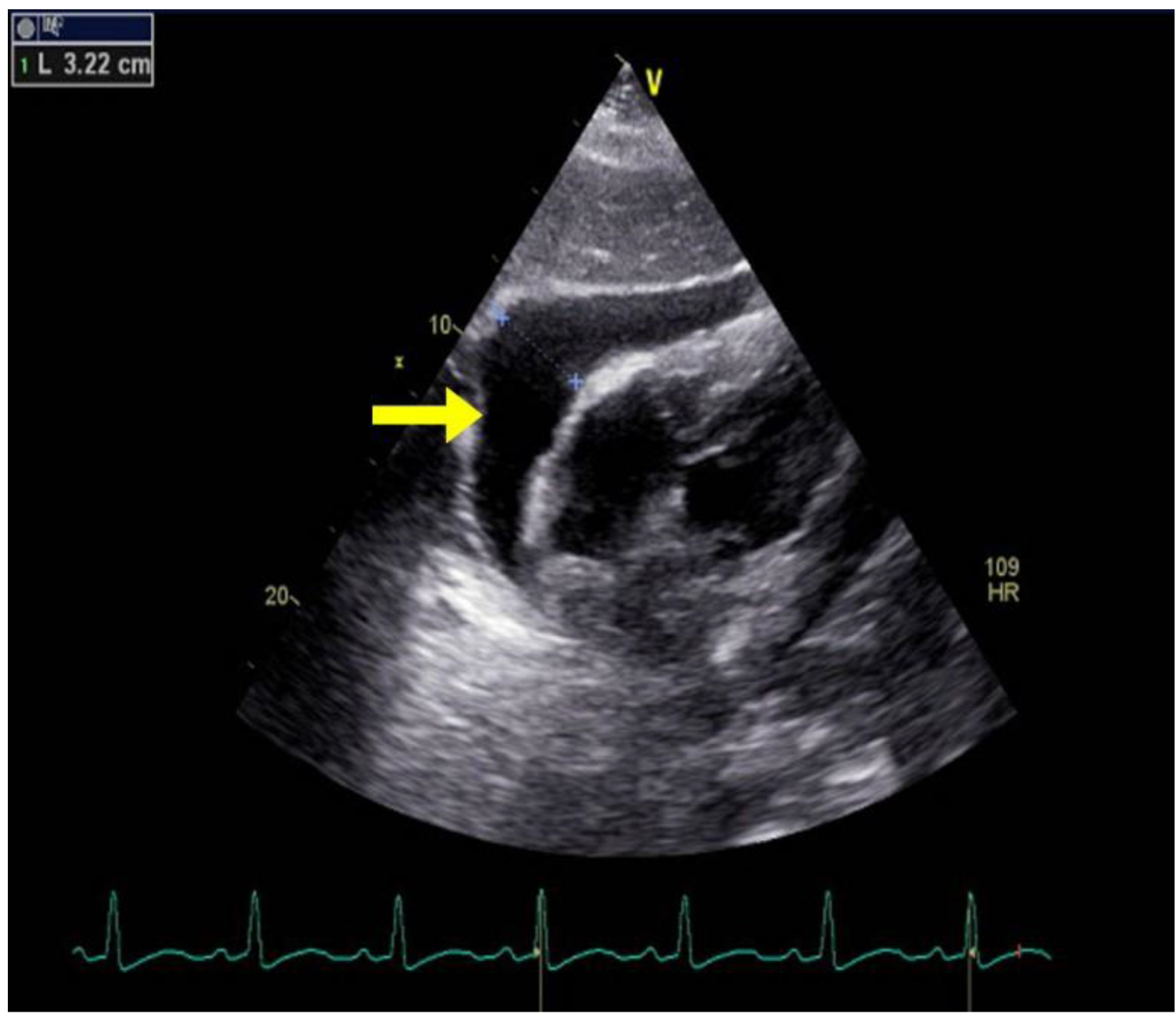

Figure 4. TTE with parasternal long axis view demonstrating large pericardial effusion measuring $3.22 \mathrm{~cm}$.

Beck's triad of hypotension, elevated jugular venous pressure, and muffled heart sounds are significant for severe cardiac tamponade, and components of this triad are seen on both our cases [4,5]. Patients often appear uncomfortable, with additional signs of cardiogenic shock including tachypnea, cool extremities, diaphoresis, and altered mental status [5]. Hypotension is typically present in acute tamponade, but some patients who initially present with subacute tamponade may be hypertensive on admission [5]. Tachycardia is frequent unless the patient is on a medication which may dampen this response [5]. Pulsus paradoxus is another hallmark of pericardial tamponade, defined by an inspiratory decrease in systolic blood pressure greater than $10 \mathrm{mmHg}$ due to a combination of a reduction in intrathoracic pressure, left ventricular stroke volume and pulse pressures $[2,6,7]$.
A differential for these symptoms includes decompensated heart failure, pulmonary embolism, pulmonary hypertension, and a right ventricular myocardial infarction [5].

EKG abnormalities in cardiac tamponade include electrical alternans and reduced voltage, which we saw in the second case. Electrical alternans is more specific, but less sensitive, and is caused by the anterioposterior swinging of the heart during systole and diastole [5]. With larger pericardial effusions, a chest x-ray may show an enlarged cardiac silhouette with a flask-like appearance [5]. The standard non-invasive method for detecting a pericardial effusion is M-mode and two- dimensional Doppler TTE. Small pericardial effusions are typical seen over the posterobasal left ventricle, which with increasing size become circumferential [5]. These are graded 
in diastole as small $(<10 \mathrm{~mm})$, moderate $(10-20 \mathrm{~mm})$, and large $(>20 \mathrm{~mm})[5,8]$. Tamponade is distinguished first by early diastolic right ventricular and late diastolic or early systolic right atrial collapse when the IPP transiently exceeds the intracavitary pressures $[5,8-$ 10]. Exaggerated interventricular size variability can be appreciated throughout the cardiac cycle, with an interventricular septal bounce towards the left ventricle [8]. IVC plethora can be seen with dilation of the IVC greater than $20 \mathrm{~mm}$, and collapse with inspiration less than $50 \%$, which has a $92 \%$ sensitivity for cardiac tamponade [8]. Changes in Doppler velocities across the mitral, tricuspid, right/left ventricular outflow tracks, and hepatic/pulmonary veins may add additional clues to this diagnosis [8]. A TTE provides better quality images, but is typically impractical to coordinate due to the need for urgent intervention. A CT or MRI may provide more details on loculated effusions and coexistent pleural effusions [5].

Definitive treatment of cardiac tamponade is through the reduction of IPP by removing pericardial fluid. In early cardiac tamponade where there is no hemodynamic compromise, conservative treatment with close hemodynamic monitoring and serial TTEs may be considered [11]. Acute cardiac tamponade is a medical emergency when hemodynamic compromise is present. Per the European Heart Journal 2015 guidelines [1,11], a pericardiocentesis is the treatment of choice, and the catheter is left in the pericardial space until the fluid return is less than $25 \mathrm{ml}$ per day [1,11]. A pericardial window is a surgical alternative requiring anesthesia, and it is used for loculated, malignant, or recurrent pericardial effusions [2,9]. An example of this procedure was seen during both of our cases. A pericardial window is also preferred if a biopsy is desired [2,9]. Intravenous hydration and rarely inotropic support or mechanical ventilation may be required depending on the clinical situation. Surgical drainage is preferred if a pericardial biopsy is needed for recurrent or loculated pleural effusions, or if a coagulopathy is present $[1,11]$. Pleural fluid studies can help identify the cause of the effusion. Post-operative monitoring includes telemetry for 24-48 hours, and a follow up TTE in 1-2 weeks and then in 6- 12 months [1].

\section{Conclusion}

Pericardial effusions can accumulate quickly resulting in acute cardiac tamponade, as seen in our first case, or in a subacute fashion with an insidious onset as evidenced by our second case. Beck's triad of hypotension, elevated jugular venous pressure, and muffled heart sounds is the classic finding of acute tamponade; however, these clinical findings may not always be present. The diagnosis is typically made by physical findings and echocardiographic evidence. It is important to establish the size and hemodynamic effect of a pericardial effusion. The first line treatment for cardiac tamponade is pericardial drainage, either by pericardiocentesis or pericardial window.

\section{Consent}

Both patients provided informed consent.

\section{Disclaimer}

This research was supported (in whole or in part) by HCA and/ or an HCA affiliated entity. The views expressed in this publication represent those of the author(s) do not necessarily represent the official views of HCA or any of its affiliated entities.

\section{Acknowledgements}

We would like to thank Dr. Hartmuth Bittner for his surgical expertise.

\section{References}

1. Leimgruber P, Klopfenstein H, Wann L, Brooks H (1983) The Hemodynamic Derangement Associated with Right Ventricular Diastolic Collapse in Cardiac Tamponade: An Experimental Echocardiography Study. Circulation 68: 612-620.

2. Vakamudi S, Ho N, Cremer P (2017) Pericardial Effusions: Causes, Diagnosis, and Management. Progress in Cardiovascular Diseases 59: 380-388.

3. Reddy P, Curtiss E, Uretsky B (1990) Spectrum of Hemodynamic Changes in Tamponade. The American Journal of Cardiology 66: 1487-1491.

4. Beck C (1935) Two Cardiac Compression Triads. Journal of the American Medial Association 104(714).

5. Reddy P, Curtiss E, O-Toole J, Shaver J (1978) Cardiac Tamponade: Hemodynamic Observations in Man. Circulation 58: 265.

6. Hoit B (2017) Pericardial Effusion and Cardiac Tamponade in the New Millennium. Current Cardiology Reports 19(57).

7. Perez-Caseres A, Cesar S, Brunet-Garcia L, Sanchez-de-Toledo (2017) Echocardiogenic Evaluation of Pericardial Effusion and Cardiac Tamponade. Frontiers in Pediatrics 5(79).

8. Adler Y, Charron P, Imazio M, Badano L, Baron-Esquivias G, (2015) 2015 ESC Guidelines for the diagnosis and management of pericardial diseases: The Task Force for the Diagnosis and Management of Pericardial Diseases of the European Society of Cardiology (ESC). European Heart Journal 36: 2921-2964.

9. Gilliam L, Guyer D, Gibson T, King M, Marshall J et al (1983) Hydrodynamic Compression of the Right Atrium: A New Echocardiographic Sign of Cardiac Tamponade. Circulation 68: 294-301.

10. Hoit B (2019) Cardiac Tamponade. In B. C. Downey (Ed.), UpToDate. Retrieved August, 8, 2019, from https://www.uptodate.com/contents/cardiactamponade\#H3809498843.

11. Zipes D, Libby P, Bonow R, Mann D, Braunwald E (2019) Braunwald's heart disease: A textbook of cardiovascular medicine (Eleventh edition). Philadelphia, PA: Elsevier.
Citation:

Kara Stout, Jennifer Forbes, Stephanie Cochran, Hussein Al-Mohamad, Merrill Krolick, Ronald Walsh (2019) Cardiac Tamponade: An Acute and Subacute Clinical Challenge. J Cardiol Clin Pract Volume 2(2): 1-6. 